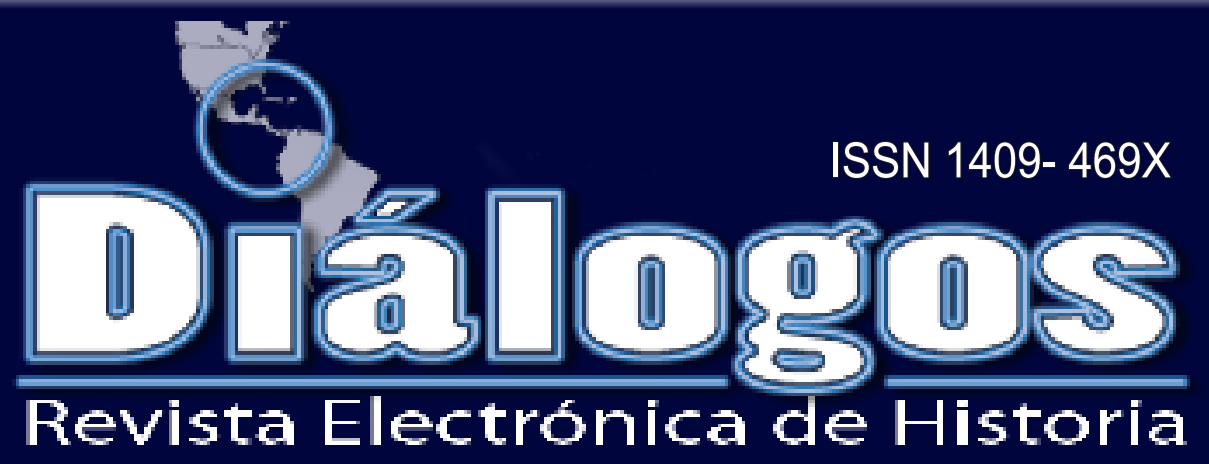

Escuela de Historia. Universidad de Costa Rica Vol. 13 No. 1 Febrero - Agosto 2012

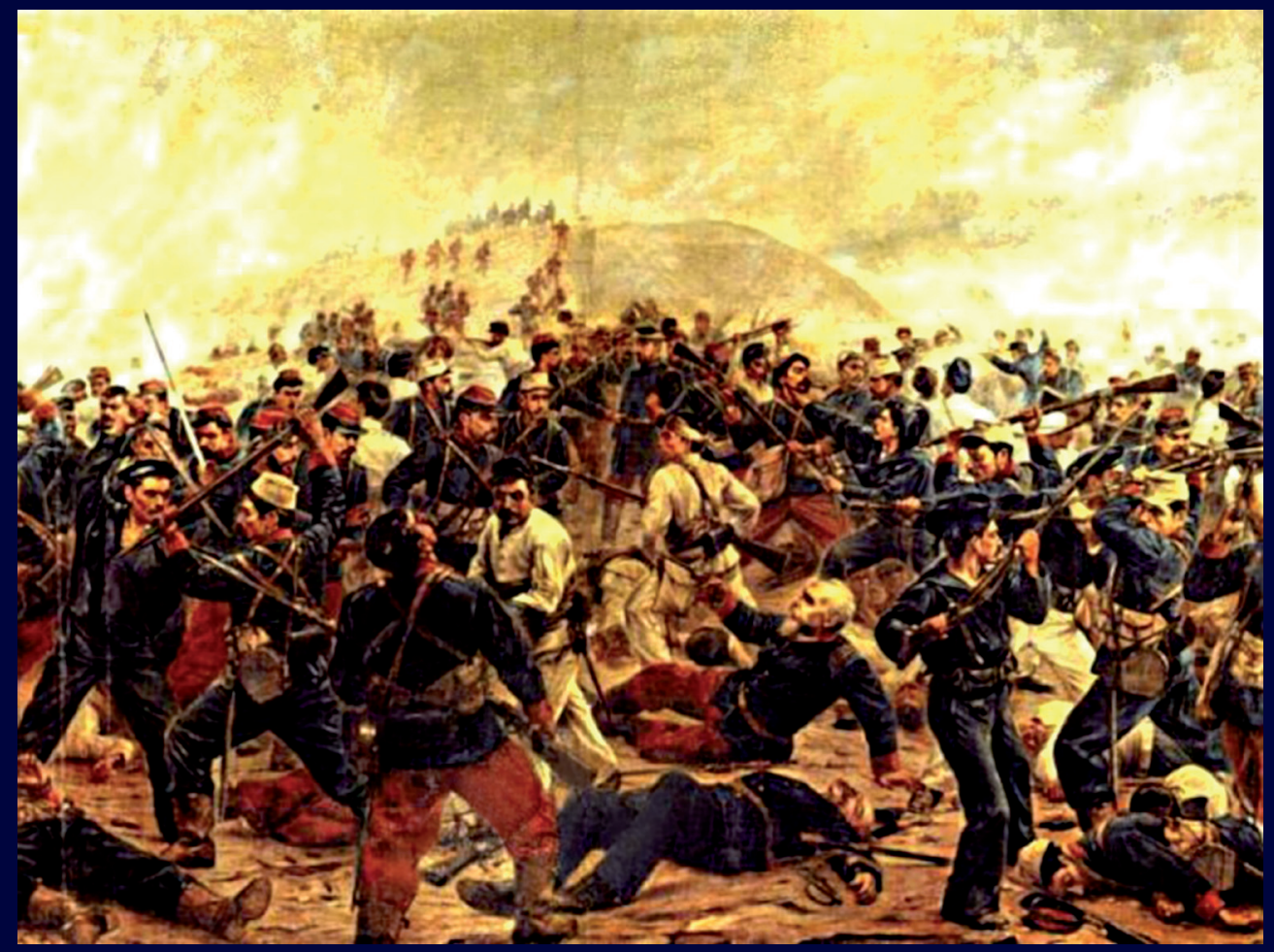

\title{
LAS BASES DE DATOS COMO HERRAMIENTA PARA LA INVESTIGACIÓN HISTÓRICA.
}

MSc. Ana Paulina Malavassi Aguilar.

Director de la Revista: Dr. Juan José Marín Hernández juan.marinhernandez@ucr.ac.cr

Editor académico: Dr. Ronny Viales Hurtado - ronny.viales@ucr.ac.cr

Editor técnico: M.Sc. Marcela Quirós G. - marcela.quiros@ucr.ac.cr Asistente: Cindy Chaves U. http://historia.fcs.ucr.ac.cr/dialogos.htm 


\section{Miembros del Consejo Editorial:}

Dr. Juan José Marín Hernández, Catedrático. Director del Centro de Investigaciones Históricas de América Central. Universidad de Costa Rica.

Dr. Ronny Viales Hurtado. Catedrático. Historia Económica y Social. Universidad de Costa Rica. Director de la Escuela de Historia. ronny.viales@ucr.ac.cr

Dr. Guillermo Carvajal. Geografía Humana. Universidad de Costa Rica.

MSc. Francisco Enríquez. Historia Social. Universidad de Costa Rica.

MSc. Bernal Rivas Especialista en Archivística. Universidad de Costa Rica.

MSc. Ana María Botey. Historia de los movimientos sociales. Universidad de Costa Rica.abotey@gmail.com

\section{Miembros del Consejo Asesor Internacional:}

Dr. José Cal Montoya. Universidad de San Carlos de Guatemala.jecalm@correo.url.edu.gt

Dr. Juan Manuel Palacio. Universidad Nacional de San Martín.jpalacio@unsam.edu.ar

Dr. Eduardo Rey. Universidad de Santiago de Compostela.ereyt@usc.es

Dr. Heriberto Cairo Carou. Departamento de Ciencia

Política y de la Administración III - Universidad

Complutense de Madrid. hcairoca@cps.ucm.es

Dra. Rosa de la Fuente. Departamento de Ciencia Política y de la Administración III Universidad Complutense de Madrid rdelafuente@cps.ucm.es

Dr. Javier Franzé. Departamento de Ciencia Política y de la Administración III Universidad Complutense de Madrid. javier.franze@cps.ucm.es

Dr. Jaime Preciado Coronado Departamento de Estudios Ibéricos y Latinoamericanos. Universidad de Guadalajara japreco@hotmail.com

Dr. Gerónimo de Sierra. Vicerrector de la Universidade Federal da Integração Latino-Americana (UNILA) y Departamento de Sociología, Facultad de Ciencias Sociales de la Universidad de la República. geronimo@fcs.edu.uy

Dr. Antonio Palazuelos. Departamento de Ciencia Política y de la Administración III - Universidad Complutense de Madrid. palazuelosa@cps.ucm.es

Dr. Werner Mackenbach. Universidad Potsdam. werner.mackenbach@uni-potsdam.de
Dr. Guillermo Castro. Ciudad del Saber Panamá. gcastro@cdspanama.org

Dra. Natalia Milanesio. University of Houston.

nmilane2@Central.UH.EDU

Dr. Ricardo González Leandri. Consejo Superior de Investigaciones Científicas - España.

rgleandri@gmail.com

Dra. Mayra Espina. Centro de Estudios Psicológicos y Sociológicos, La Habana. mjdcips@ceniai.inf.cu

Dra. Montserrat Llonch. Departamento de Economía e Historia Económica Universidad Autónoma de Barcelona montserrat.llonch@uab.es

Dra. Estela Grassi. Universidad de Buenos Aires. estelagrassi@gmail.com

Portada:

Óleo de Juan Lepiani titulado Batalla de Arica (1880-Museo de los Combatientes de Arica) : Volumen 13- No. 1, artículo 2 "Sois vosotros valientes héroes. Somos nosotros simples humanos"... de David Angelo Coronado Canales. Fuente: http://members.tripod.com/ Guerra_del_Pacifico/ARICA3. html

"Diálogos Revista Electrónica de Historia" se publica desde octubre de 1999.

\section{Diálogos está en los siguientes repositorios:}

Dialnet

http://dialnet.unirioja.es/servlet/revista?tipo_busqueda=CODIGO\&clave_revista=3325

Latindex

http://www.latindex.unam.mx/larga.php?opcion=1\&folio=12995;

REDALYC

http://redalyc.uaemex.mx/src/inicio/FrmBusRevs2.jsp?iEdoRev=2\&cvepai=11

LANIC

http://lanic.utexas.edu/la/ca/cr/indexesp.html;

Repositorio de Revistas Universidad de Costa Rica http://www.latindex.ucr.ac.cr/

Directorio y recolector de recursos digitales del Ministerio de Cultura de España

http://roai.mcu.es/es/inicio/inicio.cmd

DOJAC Directory of open access \& Hybrid journals http://www.doaj.org/doaj?func=byTitle\&hybrid=1\&query=D

Biblioteca de Georgetown

http://library.georgetown.edu/newjour/d/msg02735.html

Asociación para el Fomento de los Estudios Históricos en Centroamérica

http://afehc.apinc.org/index.php?action=fi_aff\&id=1774 
Universidad de Saskatchewan, Canadá

https://library.usask.ca/ejournals/view/1000000000397982

\section{Monografias}

http://www.monografias.com/Links/Historia/more12.shtm

\section{Hispanianova}

http://hispanianova.rediris.es/general/enlaces/hn0708.htm

Universidad del Norte, Colombia

http://www.uninorte.edu.co/publicaciones/memorias/enlaces.html

Universidad Autónoma de Barcelona

http://seneca.uab.es/historia/hn0708.htm

Repositorio Invenia - Gestión del Conocimiento

http://www.invenia.es/oai:dialnet.unirioja.es:ART0000086144

Enlace Académico

http://www.enlaceacademico.org/biblioteca/revistas-en-formato-digital-centroamerica/

\section{Electronic Resources}

http://sunzi1.lib.hku.hk/ER/detail/hkul/3987318

Revistas académicas en texto completo

http://web.prw.net/ vtorres/

\section{Diálogos se anuncia en las siguientes} instituciones y sitios académicos:

\section{Maestroteca}

http://www.maestroteca.com/detail/553/dialogos-revista-electronica-de-historia.html

\section{Biblioteca de Georgetown}

http://library.georgetown.edu/newjour/d/msg02735.htm
Asociación para el Fomento de los Estudios Históricos en Centroamérica

http://afehc.apinc.org/index.php?action=fi_aff\&id=1774

Universidad de Saskatchewan, Canadá

https:/library.usask.ca/ejournals/view/1000000000397982

\section{Monografias}

http://www.monografias.com/Links/Historia/more12.shtml

Hispanianova

http://hispanianova.rediris.es/general/enlaces/hn0708.htm

Universidad del Norte, Colombia

http://www.uninorte.edu.co/publicaciones/memorias/enlaces.html

\section{Universidad Autónoma de Barcelona}

http://seneca.uab.es/historia/hn0708.htm

Repositorio Invenia - Gestión del Conocimiento http://www.invenia.es/oai:dialnet.unirioja.es:ART0000086144

\section{Enlace Académico}

http://www.enlaceacademico.org/biblioteca/revistas-en-formato-digital-centroamerica/

\section{Electronic Resources}

http://sunzi1.lib.hku.hk/ER/detail/hkul/3987318

Revistas académicas en texto completo http://web.prw.net/ vtorres/

La revista electrónica Diálogos es financiada por la Vicerrectoría de Investigación de la Universidad de Costa Rica
Citado en Dialnet - Latindex-

Redilac- Directorio y recolector de recursos digitales del

Ministerio de Cultura de España cc) creative 



\title{
LAS BASES DE DATOS COMO HERRAMIENTA PARA LA INVESTIGACIÓN HISTÓRICA
}

\author{
Palabras claves \\ Informatización de fuentes históricas, bases de datos históricas, metodología de la investigación \\ histórica.
}

\section{Keywords}

Historical informatics, databases historical, historical research methodology.

Fecha de recepción: 3 junio de 2011 - Fecha de aceptación: 26 febrero de 2012

\section{Resumen}

El propósito de estas notas introductorias consiste en presentar someramente las bondades y las posibles limitaciones que implica el uso de bases de datos para la investigación histórica, para finalizar exponiendo, in extenso, un producto construido con base en el levantamiento de diversas fuentes primarias.

\begin{abstract}
The purpose of these introductory notes is to present briefly the advantages and possible limitations associated with using databases for historical research, to finish explaining, at length, a product built based on the lifting of various primary sources.
\end{abstract}




\title{
LAS BASES DE DATOS COMO HERRAMIENTA PARA LA INVESTIGACIÓN HISTÓRICA
}

\author{
MSc. Ana Paulina Malavassi Aguilar
}

Las fuentes de las que se nutre la investigación histórica son muy variadas, pudiéndose resumir en todas las manifestaciones culturales que han producido los diversos grupos sociales desde la aparición de la humanidad, y que son tanto tangibles como intangibles.

A lo largo del tiempo el ser humano ha utilizado diversos medios para preservar la información, entre ellos, la piedra, la madera, las tablillas de arcilla, el papiro, el pergamino, el papel y, más recientemente, los medios electrónicos. Estos últimos adquieren considerable importancia, dada su capacidad de almacenar grandes cantidades de información, así como de garantizar mayor facilidad de transporte y de consulta - gracias al uso de discos ópticos, memorias y discos duros portátiles-y de democratizar el acceso a la información por medio de la publicación de materiales en la Web y del envío de información a través del correo electrónico, favoreciendo un tráfico de datos sin precedentes en la historia de la humanidad.

$\mathrm{Y}$ es que el ordenador y todos los usos que el mismo permite, aunado a la revolución en la información, potenciada por la creación de la Web, han venido a enriquecer sensiblemente el utillaje del historiador, gracias al acceso-en algunas ocasiones gratuito-a una cantidad infinita de artículos, imágenes y audios de interés académico, así como a las clásicas fuentes de archivo, biblioteca o hemeroteca digitalizadas. A estos acervos debe agregarse la profusión de materiales electrónicos que diariamente se producen en diferentes instituciones públicas y privadas, que han contribuido a engrosar sensiblemente los caudales documentales del historiador.

Actualmente existe una multitud de bases de datos de texto completo y de datos referenciales, algunas gratuitas y otras por suscripción, que posibilitan a los científicos sociales estar al tanto de las últimas tendencias en el campo de trabajo de su interés. Entre las bases de texto completo más grandes, prestigiosas y privadas, que comprenden diferentes áreas del conocimiento, se pueden citar JSTOR, Project Muse, ProQuest y EBSCO. Entre las bases públicas, aunque de menores dimensiones, resaltan DOAJ, E-Revist@s, NSDL y Redalyc.

La digitalización de las fuentes históricas ha asumido diversas facetas, entre las más complejas y pretensiosas exalta el proyecto de digitalización de documentos de los archivos españoles, ${ }^{1}$ que brinda la posibilidad de consultar en forma directa una amplia gama de ficheros históricos, y que en el futuro pretende convertirse en el Portal Iberoamericano de Archivos. ${ }^{2}$ Indudablemente la digitalización es sinónimo no sólo de difusión sino también de preservación, porque ha abierto grandes perspectivas "para el uso de un material que a menudo ha estado expuesto 
a factores climáticos, hongos y a la voracidad de los insectos." ${ }^{3}$ Factores a los que es necesario sumar el desgaste y deterioro provocados por la manipulación de los usuarios, así como por el uso de métodos de reproducción tan dañinos como el fotocopiado o la microfilmación. Lo último es perfectamente subsanable con el empleo de la fotografía digital, la cual-por razones de lucro o por erróneas políticas de conservación - no siempre es autorizada en todos los repositorios de información.

Entre las iniciativas quizás menos pretensiosas, pero no por ello menos importantes, está la creación y difusión de bases de datos históricas, las cuales conceptualizamos como información de carácter histórico respaldada electrónicamente, lo cual equivale a un conjunto de datos lógicamente relacionados entre sí que se pueden resguardar en diversos medios digitales, y que responden a un eje temático claramente definido y que puede ser consultado por más de un usuario dependiendo de los niveles de restricción impuestos. El ejemplo más modesto que se puede mencionar es la base de datos que aquí se presenta, y uno de los más presuntuosos es la base de datos privada FICHOZ. ${ }^{4}$

Existen diversos tipos de bases de datos, sin embargo, los que mejor se acoplan a la investigación histórica son la tabla única o base sencilla, así como el conjunto de tablas relacionadas entre sí llamado bases relacionales, optar por uno u otro tipo depende del nivel de dificultad y de la complejidad de la investigación. Las bases de datos sencillas consisten en tablas donde se almacena la información en una única tabla, la cual está compuesta por un conjunto de filas y de columnas, las primeras son los registros y las segundas los atributos particulares de cada registro. Las bases de datos relacionales almacenan todos los datos en diversas tablas y la relación entre ellas se efectúa a partir de los datos coincidentes entre las tablas.

Las bases de datos históricas bien constituidas reúnen una serie de características que facilitan considerablemente el trabajo del historiador. Dentro de esas particularidades resaltan:

1. Unificación de la información, en ciertos casos procedente de variados focos documentales, en torno a un eje temático específico, ya que una base responde al planteamiento de una problemática muy específica, donde las diversas entradas permiten medir las variables de la investigación a través de sus respectivos indicadores

2. Automatización y agilización en la consulta de los datos

3. Maleabilidad al posibilitar la introducción de cambios en la estructura de los datos y la actualización de los mismos

4. Depuración de los datos con la consecuente eliminación de las duplicaciones

La base de datos que a continuación se presenta, una tabla única, ha sido construida a partir de las referencias recabadas en un formulario, que fue creado con el propósito de levantar las huellas de carácter prosopográfico dejadas en los 
expedientes judiciales por un grupo de sujetos tachados por las autoridades y por algunos de sus coetáneos como ebrios, jugadores y vagos, lo cual da cabida a una amplia maraña de deslices. Los datos han sido recopilados con base en un muestreo efectuado en el fondo judicial del Archivo Nacional de Costa Rica para el período 1775-1850, y por tratarse de un muestreo la base queda abierta para ser alimentada con nueva información en el futuro.

Dicha base se caracteriza por utilizar un modelo de matriz rectangular donde cada columna representa un atributo diferente asociado a un dominio de valores, en tanto que cada fila de registros hace alusión a una entidad o caso en particular y como tal se puede manejar con independencia del resto. El banco de datos ha sido construido en Excel.

Los campos que sustentan cada uno de los registros de la base de datos "Marginales" son catorce en total. Los primeros siete registros permiten formarse una idea general sobre el imputado, es decir, su apellido, nombre y sexo, seguido de su lugar de nacimiento y de residencia, así como del oficio que reporta. Los siguientes seis registros recogen las faltas imputadas con base en su orden de aparición en el expediente judicial. El último registro indica el año o la fecha exacta en la que se levanta la causa judicial.

Como punto débil se debe señalar que esta base ha sido construida con un fin específico-manejar un panorama general sobre los marginales - por lo que el levantamiento de la información ha sido selectivo, fruto de un proceso de discriminación y edición, quedando en los expedientes datos muy valiosos para investigaciones con otros enfoques. Este detalle nos permite advertir la importancia de construir bases de datos a texto completo, donde el investigador pueda tener una visión de conjunto, ya que la interpretación de los datos no puede darse independientemente del contexto del cual se toman.

La utilización sistemática de la informática-no del simple uso del procesador de texto y de la red informática mundial-en el quehacer del historiador no ha estado exenta de críticas. Una falsa creencia ha sido la afirmación de que solamente la información de carácter cuantitativo es susceptible de ser procesada en un ordenador, relegándose lo cualitativo a la lógica interpretativa del historiador. ${ }^{5}$ Si bien la lógica interpretativa nunca está de más, existen programas informáticos aptos para desarrollar uno u otro procesamiento de los datos. En este sentido, otra debilidad de la base es su carácter eminentemente cuantitativo, aunque una lectura diferente de la documentación y el empleo del software adecuado puede conducir a un estudio cualitativo lo suficientemente provechoso.

En síntesis, la informática es más que un corolario a la investigación histórica de base, es una herramienta fundamental cuya explotación en gran escala se prevé como un medio imprescindible para organizar, almacenar, diseminar y preservar los datos. 


\section{CITAS Y NOTAS}

1 Portal de Archivos Españoles (PARES), http://pares.mcu.es/.

2 "México será sede del I Congreso de la Cultura Iberoamericana en 2008", Portal Iberoamericano de Gestión Cultural, http://pgcdes.ird.ub.es/gc/event/detailEvent. jsp?idEvent $=158912 \&$ \&idSection $=57823$.

3 Porfirio de Cruz Samudio, "Uso de la informática en la investigación histórica", Revista Cultural Lotería, $\mathrm{N}^{\circ}$. 460, mayo-junio 2005, p.22.

4 La base FICHOZ ha sido creada por el grupo PAPE (Personal Administrativo y Político español del siglo XVIII) y registra en archivos interrelacionados las carreras vitales de miles de personajes de la historia moderna. Jean Pierre Dedieu, "Les grandes bases de donneés: une nouvelle approche de l'histoire sociale. Le système Fichoz", Revista da Facultade de Letras História, serie III, vol. 5, 2004, pp. 101-114.

5 Un buen ejemplo de este tipo de razonamiento lo constituye la siguiente cita: "cualquier máquina puede procesar el número de hijos por matrimonio y hallar, sin mayor dificultad, gracias a una base de datos diseñada a tal efecto, la media de hijos de cada unión conyugal. Sin embargo, es imposible que éste pueda evaluarnos los resultados de cualquier política matrimonial o los intereses ocultos que subyacen a la alianza entre linajes, a veces, incluso totalmente incomprensibles para el marco teórico que la Historia de la Familia nos proporciona. Es ahí donde la explicación individual de cada historiador dota de sentido a tan complejo problema." Raúl Molina Recio, "De la utilidad y los inconvenientes de la informática para la historia", Tiempos modernos: Revista Electrónica de Historia Moderna, №. 7, 2002-2003, http://www.tiemposmodernos.org/floridablanca/resumenmolina.htm.

\section{ACERCA DE LA AUTORA}

MSc. Ana Paulina Malavassi Aguilar: Universidad de Costa Rica Firma Docente Escuela de Historia. Máster en Historia, se desempeña como docente en la Escuela de Historia, en la Escuela de Estudios Generales y en el Posgrado Centroamericano en Historia, y como investigadora en el Centro de Investigaciones Históricas de América Central, entes todos adscritos a la Universidad de Costa Rica. 